\title{
O Pensamento Computacional em Atividades de Ensino mediadas pelo Professor do Ensino Fundamental I: Um Estudo de Caso
}

\author{
Suéllen Rodolfo Martinelli ${ }^{1}$, Luciana Aparecida Martinez Zaina ${ }^{1}$, \\ Tiemi Christine Sakata ${ }^{1}$
}

\author{
${ }^{1}$ Departamento de Computação de Sorocaba - Universidade Federal de São Carlos \\ CEP 18052-780 - Sorocaba - SP - Brasil
}

\begin{abstract}
This paper presents a case study on educational practices that were applied to support the development of Computational Thinking $(C T)$ in children. Teachers who belong to public Elementary School elaborated and conducted activities with the aim of introducing the CT to their students. We provided continuing education course for these teachers and collected data to carried out the qualitative analysis based on Grounded Theory. The results indicate three different formats of activities that can encourage the development of Computational Thinking skills in students of elementary schools. These skills match to those cited in Brazil's BNCC document.
\end{abstract}

Resumo. Este trabalho apresenta um estudo de caso sobre práticas educativas voltadas a desenvolver o Pensamento Computacional (PC) em crianças. Essas atividades foram construídas e mediadas por docentes que atuam no Ensino Fundamental I de escolas públicas. Com a aplicação de uma formação continuada para os professores participantes, foram coletados dados qualitativos e conduzida uma análise por meio da Teoria Fundamentada. Os resultados indicam três diferentes formatos de atividades que podem estimular o desenvolvimento das habilidades do PC nos alunos do ensino básico. Essas habilidades são apontadas também na Base Nacional Comum Curricular (BNCC).

\section{Introdução}

O termo Pensamento Computacional (PC), do inglês Computational Thinking, foi difundido na academia a partir de 2006, por Jeannette Wing [Wing 2006]. A pesquisadora esclarece que é possível utilizar os conceitos e habilidades fundamentais que sustentam a Ciência da Computação para interpretar e resolver problemas de todas as áreas do conhecimento. O PC trata da capacidade de se pensar de forma abstrata, reduzir problemas em partes menores e mais simples de se resolver, sem obrigatoriamente ser preciso a aplicação de técnicas de programação ou de meios digitais para esse fim [Wing 2014].

Na literatura, não há um consenso sobre quais são as concepções que caracterizam o raciocínio computacional [Kalelioglu et al. 2016]. Porém, estes estão em comum acordo em reconhecer que esse "pensar computacional" precisa ser uma habilidade de todos e para todos. O ensino de conceitos básicos de Computação nas escolas é fundamental, assim como a sua inserção no atual currículo brasileiro por meio de políticas nacionais, possibilitando a formação de cidadãos capazes de viverem em uma sociedade globalizada [Silva et al. 2017]. Para isso, é vital promover a formação continuada de professores do 
VII Congresso Brasileiro de Informática na Educação (CBIE 2018)

Anais do XXIV Workshop de Informática na Escola (WIE 2018)

Ensino Básico para gerar investigações quanto à disseminação e avaliação das habilidades ligadas ao PC para a sua efetiva aplicação nas instituições de ensino [Valente 2016].

Este artigo tem como objetivo apresentar um estudo de caso que investigou práticas educativas voltadas ao desenvolvimento do PC em crianças, sendo tais atividades construídas e mediadas por docentes do Ensino Fundamental I (EFI) de instituições públicas. O estudo de caso foi conduzido sob uma perspectiva exploratória cujo foco foi verificar a é possível inserir o PC em atividades de ensino direcionadas ao EFI e como esse raciocínio foi conduzido em práticas educacionais pelos professores. Para esse fim, foi realizado um curso de formação continuada para que os docentes compreendessem as habilidades do PC para atuarem no planejamento e condução de atividades que fomentem tais habilidades nas crianças. A partir dos dados coletados foi realizada uma análise qualitativa dos dados apoiada na Teoria Fundamentada [Charmaz 2009]. Entre as práticas criadas pelos participantes, foi possível extrair três formatos (padrões) de atividades de ensino que potencializam o desenvolvimento de habilidades do PC.

O artigo está estruturado como segue: a Seção 2 apresenta discussões e trabalhos relacionados; a Seção 3 fornece a metodologia utilizada no estudo de caso; a Seção 4 discute os processos de avaliação, resultados obtidos e reflexões acerca dos formatos das atividades que foram encontradas durante a análise; a Seção 5 responde as perguntas de pesquisa por meio de confirmação de hipóteses; a Seção 6 realiza as considerações finais.

\section{Fundamentação Teórica e Trabalhos Relacionados}

Atualmente, o Pensamento Computacional é entendido como um conjunto de habilidades essenciais à formação do indivíduo, para capacitá-lo na resolução de problemas diversificados, sendo seu domínio tão importante quanto ao da leitura, da escrita e da aritmética [Wing 2014]. Percebendo a relevância do tema, países como Austrália, Estados Unidos, Canadá e Reino Unido promovem o ensino de Computação em suas escolas desde o Ensino Básico [França and Tedesco 2015]. Organizações como a Computer Science Teachers Association (CSTA), a Association for Computing Machinery (ACM) e a International Society for Technology in Education (ISTE) compuseram normativas difundidas pelo mundo destinadas ao ensino de Computação e do Pensamento Computacional.

Além disto, existe a difusão de iniciativas, no qual produzem e distribuem com exemplos e materiais didáticos gratuitos sobre como conduzir atividades que estimulem esse raciocínio computacional nas crianças. Algumas das iniciativas desses países são: o projeto britânico Computing At School ou CAS (https://www.computingatschool.org. uk), o CS Unplugged (http://csunplugged.org/) da Nova Zelândia, a plataforma Code.org (https://studio.code.org), e a formação online Exploring Computational Thinking (https: //edu.google.com), para docentes, da Google for Education.

A iniciativa Barefoot (https://barefootcas.org.uk) foca na produção de materiais e na realização de treinamentos para os professores do Ensino Fundamental I, sendo este, o mesmo contexto deste estudo. Baseando-se em suas definições, as concepções do PC consideradas neste trabalho são: o raciocínio lógico, que auxilia a analisar fatos; a ideia de algoritmo, que exige uma sequência precisa de instruções para executar uma tarefa; a decomposição, capacidade de quebrar um problema em partes menores e gerenciáveis; a percepção de padrões, para ser capaz de reconhecer similaridades entre problemas; a abstração, habilidade que ajuda na sintetização dos fatos para decidir o que é importante; 
VII Congresso Brasileiro de Informática na Educação (CBIE 2018)

Anais do XXIV Workshop de Informática na Escola (WIE 2018)

e a avaliação, capacidade de julgar com base em fatos, auxiliando na tomada de decisões.

No Brasil, a Sociedade Brasileira de Computação (SBC) estimula projetos sobre Computação e/ou Pensamento Computacional no Ensino Básico, sendo alguns grupos responsáveis por realizar intervenções, oficinas e cursos com a comunidade. Das iniciativas ${ }^{1}$ mantidas por universidades, destacam-se: o Meninas Digitais, o Computação na Escola, o Laboratório de Inovação Tecnológica na Educação (LITE) e o Computing to You! (C2Y!).

Aliado ao crescente número de pesquisas sobre práticas educacionais direcionadas ao PC no contexto brasileiro [Ferreira et al. 2015, Barcelos et al. 2016, de Melo Reis et al. 2017, Raabe et al. 2017, Silva et al. 2017], a SBC também têm discutido com representantes da Base Nacional Comum Curricular (BNCC), sobre a alteração da base para adequar um currículo voltado ao desenvolvimento do PC em seu universo e cultura digital. Esse debate é significativo, uma vez que a atual versão da BNCC (http://basenacionalcomum.mec.gov.br) aborda o PC de forma estrita, integrando-o apenas à Matemática, e não como área, como orienta a SBC [Leite and da Silva 2017].

Diante dos desafios e oportunidades apontadas por [França and Tedesco 2015, Valente 2016] para contribuir na integração de PC no Ensino Básico brasileiro, destacamse alguns trabalhos relacionados. Entre eles, relatos de experiência sobre o tema, como: a condução de Atividades Desplugadas interdisciplinares sobre Computação, entre alunos do Ensino Fundamental II e do Ensino Médio [Ferreira et al. 2015]; e a aplicação de um curso para estudantes de $8^{\circ}$ e $9^{\circ}$ ano com foco no desenvolvimento de jogos eletrônicos, a partir de abordagens como programação e Storytelling [de Melo Reis et al. 2017]. Também há alguns estudos que se enquadram na capacitação docente como a formação inicial e continuada sobre PC, na modalidade Ensino à Distância, dedicada apenas aos professores de Matemática [Barcelos et al. 2016]; e a formação presencial sobre PC, ofertada aos docentes de escolas públicas de Recife-PE [Silva et al. 2017].

Esta investigação diferencia-se das citadas acima por oferecer suporte aos professores do Ensino Fundamental I para que eles possam incluir o PC em suas práticas pedagógicas. Além disso, os resultados deste estudo são suportados por uma avaliação qualitativa, sendo essa incorporada de maneira secundária em investigações direcionadas à Computação. Assim, espera-se com esta pesquisa, contribuir em aspectos que fazem parte do planejamento de atividades de ensino que estimulem o PC nos alunos.

\section{Metodologia do Estudo de Caso}

Por não existir obrigatoriedade na implantação de currículos específicos ou, pelo menos, de práticas que estimulem o PC, essas atividades acabam acontecendo somente por mediação de um profissional da Computação, quando a instituição possui o mesmo. Ao considerar esse contexto e a infraestruturas das escolas no país, tem-se como motivação a tentativa de capacitar o próprio docente do Ensino Básico, para que atue como elaborador e mediador de atividades educacionais sobre PC, de acordo com a sua realidade institucional. Assim, esta pesquisa se propõe a investigar as seguintes questões:

Pergunta 1 (P1): Como é possível incluir o Pensamento Computacional em Atividades de Ensino direcionadas ao Ensino Fundamental I?

\footnotetext{
${ }^{1}$ Para conhecer essas e outras iniciativas, acesse: http://www.sbc.org.br/institucional-3/chancela-sbc/.
} 
VII Congresso Brasileiro de Informática na Educação (CBIE 2018)

Anais do XXIV Workshop de Informática na Escola (WIE 2018)

Pergunta 2 (P2): Como o docente trata/conduz as habilidades do Pensamento Computacional em Atividades de Ensino direcionadas ao Ensino Fundamental I?

Para responder as perguntas foi planejado e conduzido um estudo de caso de caráter exploratório [Lazar et al. 2017]. Os dados coletados foram analisados de forma qualitativa. O Estudo de Caso foi efetuado conforme a descrição da Figura 1.

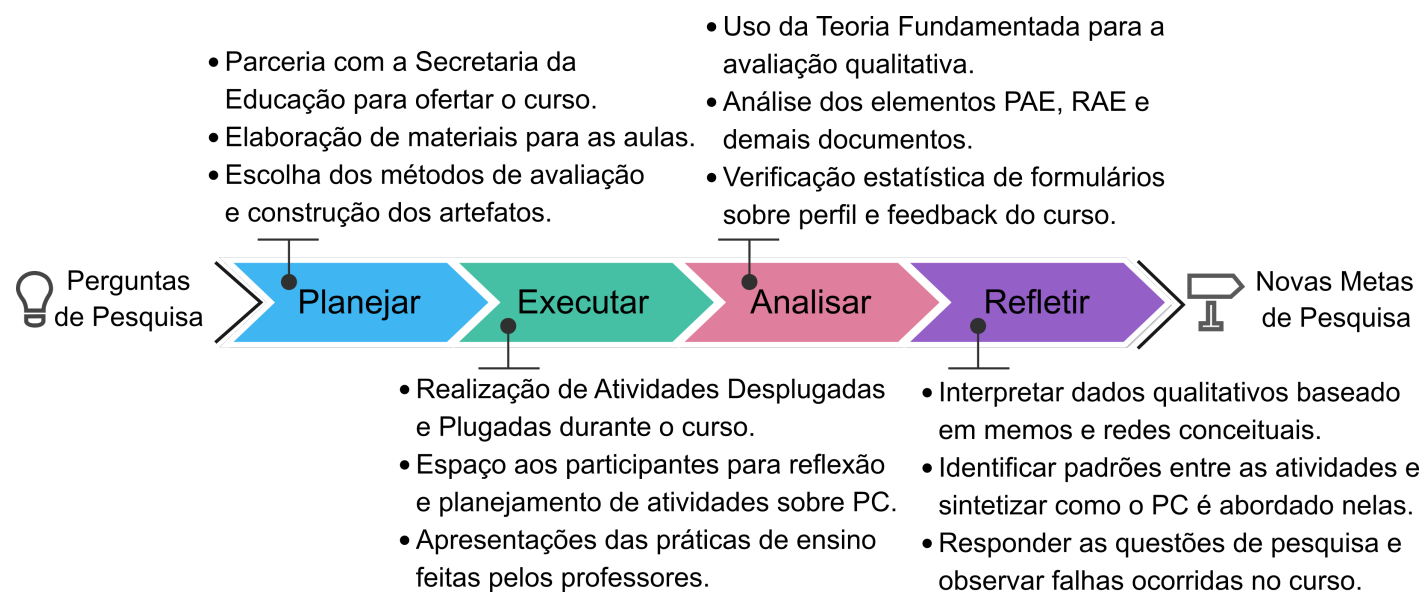

Figura 1. Visão geral da metodologia: métodos, artefatos e ações.

A fase de planejamento teve o objetivo de definir uma formação continuada sobre PC para profissionais do EFI, incluindo a elaboração de materiais e métodos de avaliação dos artefatos propostos. Nesta fase, foram concebidas apresentações eletrônicas para as aulas e artefatos destinados à coleta de dados, disponíveis em http://bit.ly/T2_WIE2018.

Para viabilizar a execução, foi firmada uma parceria entre a UFSCar e a Secretaria de Educação de Salto de Pirapora, por meio de uma Atividade de Extensão ( $\mathrm{n}^{\mathrm{o}}$ 23112.000818/2017-17). A Figura 2 trata da organização do curso ministrado, que pontua o que foi aplicado em cada aula e as habilidades do PC abordadas. Com duração de 120 minutos cada, as aulas foram ministradas em encontros semanais e/ou quinzenais, alocadas de acordo com a disponibilidade dos participantes. Todas as aulas aconteceram na EMEF Prof. Roberto Marcello, durante o primeiro semestre de 2017.

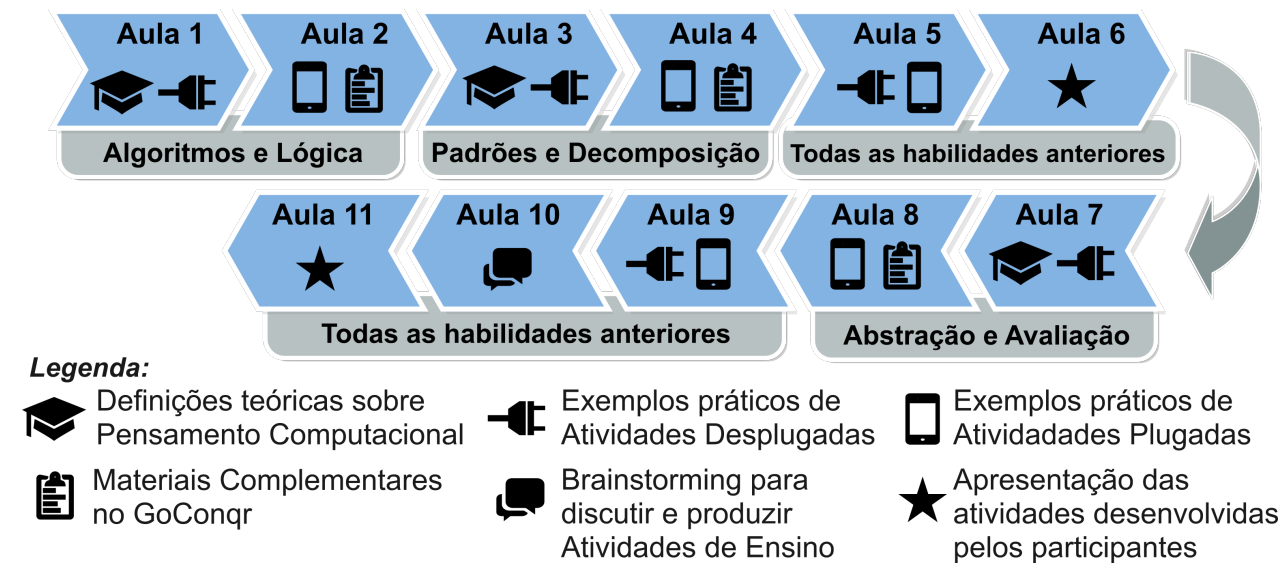

Figura 2. Aulas e organização dos conceitos de Pensamento Computacional abordados na Formação Continuada (Curso de Extensão). 
VII Congresso Brasileiro de Informática na Educação (CBIE 2018)

Anais do XXIV Workshop de Informática na Escola (WIE 2018)

Durante a execução os conceitos do PC foram introduzidos em pares e de forma incremental, por meio de atividades relacionadas a assuntos de um dado componente curricular. Entre as práticas conduzidas com os professores, destacam-se as Atividades Desplugadas, caracterizadas por não usarem nenhum meio digital para abordar o PC, e as Atividades Plugadas, realizadas no laboratório de informática da instituição em que o curso foi aplicado. Além dos computadores usados nas Atividades Plugadas, foram empregados nessas práticas 9 tablets emprestados pelo Instituto Federal de Educação, Ciência e Tecnologia de São Paulo (IFSP Câmpus Itapetininga).

A formação iniciou com 41 docentes do EFI da rede municipal de educação de Salto de Pirapora-SP, sendo que desses, 14 concluíram o curso. Os dados considerados neste estudo são apenas dos professores que finalizaram o curso, tendo a maioria deles idade entre 31 e 40 anos, com presença predominante de profissionais do sexo feminino. Aos 54,5\% dos participantes, o tempo de experiência é entre 6 e 10 anos de docência, e 63,5\% elencaram ter mais dificuldade em lecionar temas da disciplina de Matemática.

As atividades de ensino sobre PC foram produzidas pelos docentes em duplas ou individual, em que estes escolheram livremente um assunto de um componente curricular, com o intuito de estimular o raciocínio computacional nas crianças em suas práticas de ensino. Tais práticas foram documentadas em dois momentos: antes da condução, em formato de planilha denominada PAE (Planilha de Dados Primários), para anotar o planejamento e; após a condução, no roteiro RAE (Roteiro da Atividade de Ensino), para documentar os procedimentos adotados na prática desenvolvida e pontuar a relação observada entre a atividade feita e os conceitos do PC estimulados. As práticas documentadas pelos professores foram aplicadas por eles em suas respectivas salas de aula. A Figura 3 ilustra as fases de aprendizagem de PC, planejamento e condução da atividade.
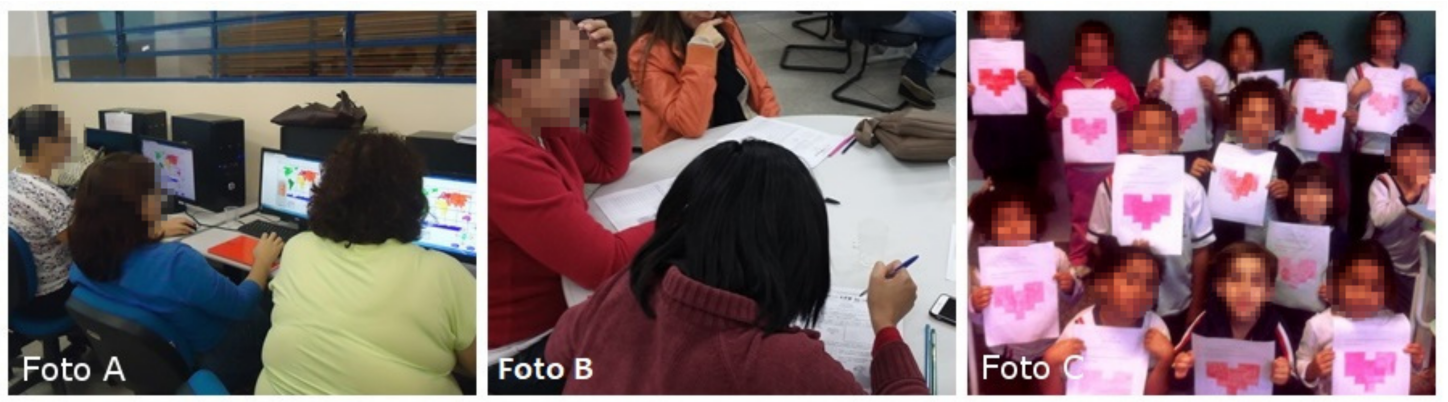

Figura 3. Docentes em Atividade Plugada (Foto A) e articulando uma prática sobre PC (Foto B). A Foto $C$ mostra a condução de uma atividade com crianças.

A fase de análise teve como objetivo reconhecer padrões quanto ao formato das atividades de ensino que buscam estimular o PC e identificar como esse raciocínio computacional é abordado nas práticas dos docentes. A análise qualitativa foi guiada pelo método da Teoria Fundamentada, ou em inglês, Grounded Theory [Bandeira-de Mello and Cunha 2003]. A intenção desse tipo de investigação é gerar uma pesquisa fundamentada a partir dos dados coletados junto aos participantes que experimentaram de um determinado processo, e assim, conceber ou descobrir uma teoria. A técnica exige que o pesquisador aprofunde-se na análise por meio de sucessivas comparações entre os dados. Os resultados vão se solidificado gradualmente. O processo foi guiado por três níveis da Teoria Fundamentada. 
VII Congresso Brasileiro de Informática na Educação (CBIE 2018)

Anais do XXIV Workshop de Informática na Escola (WIE 2018)

No Nível 1 (Codificação Inicial) foi empregada a Codificação Aberta, técnica em que o pesquisador examina os dados e cria rótulos em fragmentos dos dados com códigos que lhes dêem significado. Não se utiliza de um conjunto pré-definido de códigos, pelo contrário, os códigos emergem dos dados empíricos. Considerou-se nesse processo todos os artefatos preenchidos pelos participantes, os 26 documentos de registro das atividades PAE e o RAE, além de outros materiais fornecidos pelos docentes, como fotos da execução das atividades e testes escritos aplicados com os alunos. $\mathrm{O}$ segundo processo neste nível foi a Codificação Focalizada, para sintetizar e explicar segmentos maiores de dados, reconhecendo os rótulos mais significativos e/ou frequentes nos dados, que permitiam uma compreensão analítica mais precisa [Charmaz 2009, p.87]. Como resultado desta etapa foram efetuadas a composição de memorandos (memos) e comentários sobre as decisões tomadas durante a análise, que compõe as anotações do avaliador.

Em seguida, foi realizada a Codificação Axial (Nível 2) aplicando-se a Comparação Incidente por Incidente. Esta técnica consiste em realizar uma comparação teórica para buscar possíveis correlações entre casos [Charmaz 2009, p.80] (ou seja, as próprias atividades de ensino produzidas pelos docentes). Para efetivar o processo de comparação, tais casos foram comparados também com as anotações do pesquisador e os respectivos documentos primários: (i) a BNCC; (ii) o CSTA K-12 Computer Science Standards e a Computational Thinking Leadership Toolkit (First Edition), ambos da CSTA (https://www.csteachers.org); (iii) o The Computational Thinker: Concepts and Approaches, da Barefoot; e (iv) a Computação na Educação Básica, da SBC (http://www.sbc.org.br). As comparações resultaram em redes conceituais que fundamentam os principais padrões e processos que fazem parte das atividades de ensino sobre PC, sendo uma dessas redes apresentada na Seção 4.

Por fim, na Codificação Seletiva (Nível 3), realizou-se uma Amostragem Teórica para "entrelaçar novamente a história fragmentada" [Charmaz 2009, p.94], com o objetivo de classificar cada atividade em uma ou mais proposições (afirmações). Essas proposições retratam padrões identificados entre as atividades de ensino e os demais documentos analisados, atuando como rótulos de agrupamentos desses casos, dando base às respostas das questões de pesquisa. Por fim, efetuou-se o Refinamento da Teoria, sendo o pesquisador capaz de inferir um esquema teórico principal [Bandeira-de Mello and Cunha 2003]. Essa etapa incluiu a conexão de hipóteses junto às perguntas de pesquisa e a construção de modelos gráficos dos formatos das atividades delimitadas (abordados na Seção 4).

Os procedimentos de cada nível de codificação, desprendeu, em média, um total de 15 horas por semana, em um período de 5 meses. A organização destes processos foram feitos no software ATLAS.ti (http://atlasti.com), sendo possível obter/reunir os respectivos elementos durante a avaliação qualitativa: (i) 57 Documentos Primários: documentos PAE, RAE, fotos enviadas por docentes e normas sobre o ensino do PC; (ii) 77 Códigos: agrupamentos de dados aplicados pelo pesquisador (iii) 43 Notas Analíticas: memos e comentários que descrevem o histórico de interpretação e fundamentam os resultados das codificações; e (iv) 7 Categorias: derivado de comparações entre códigos e notas analíticas, definido por um conceito e associado aos padrões vistos nas atividades. 
VII Congresso Brasileiro de Informática na Educação (CBIE 2018)

Anais do XXIV Workshop de Informática na Escola (WIE 2018)

\section{Resultados e Discussões}

A Figura 4 mostra o mapa conceitual, que foi um dos resultados obtido após a análise dos dados. A rede expõe a síntese das principais categorias e conceitos, sendo estas, unidades elementares identificadas quanto à inclusão e condução do PC nas práticas educacionais. Outros detalhes da análise e resultados obtidos encontra-se em [Martinelli et al. 2018].

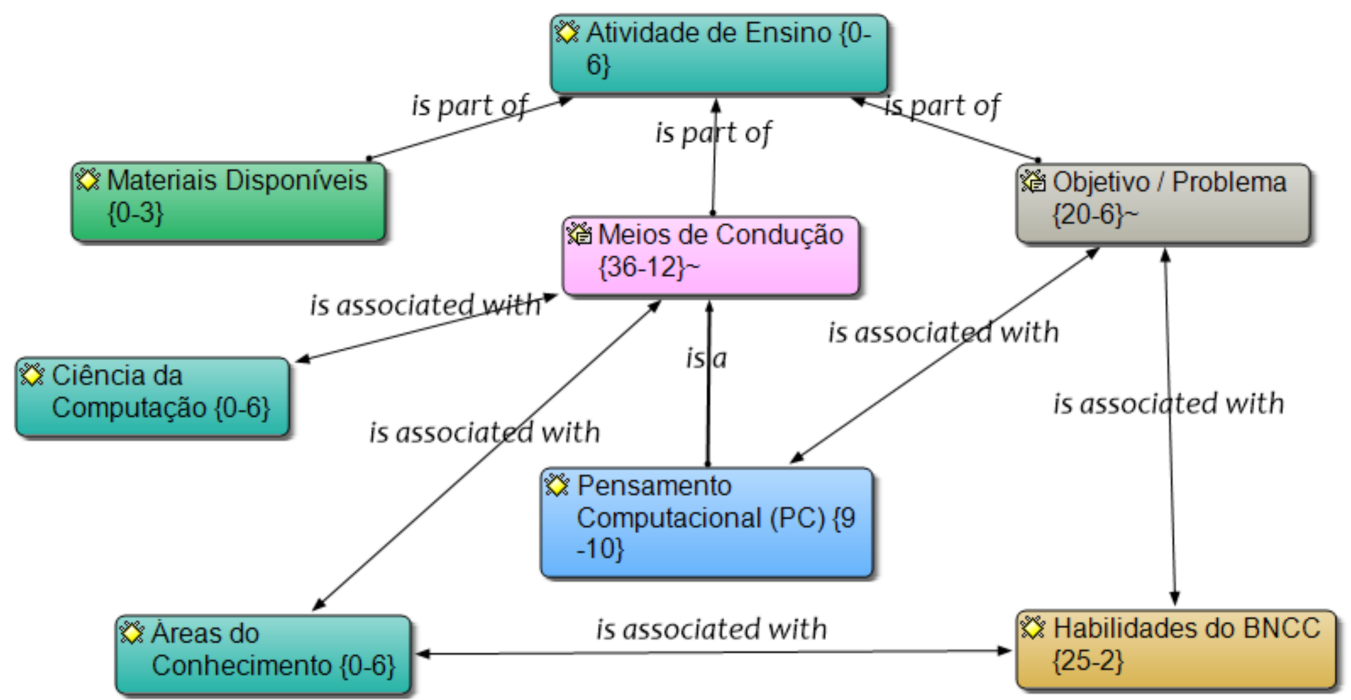

Figura 4. Mapa conceitual com unidades elementares para inclusão e condução do PC nas práticas educacionais.

O mapa conceitual expõe as subcategorias relacionadas à primeira categoria Atividade de Ensino, agente que promove um contexto de ensino-aprendizagem baseado em um problema a ser resolvido, constituído por três elementos essências: Objetivo / Problema, que reúne habilidades da BNCC e do Pensamento Computacional; Materiais Disponíveis, que expressam insumos e recursos aplicados pelos docentes nas atividades; e Meios de Condução, que classifica abordagens e procedimentos de como as práticas foram conduzidas e/ou mediadas. No Áreas do Conhecimento há a concatenação por área de conhecimento dos temas e habilidades identificadas nas atividades. Essas habilidades são agrupadas no código Habilidades do BNCC, que reuniu 18 habilidades detectadas, sendo elas agregadas aos componentes curriculares de Língua Portuguesa, Artes, Matemática, Ciências e Geografia. Esse resultado difere do que é exposto pela BNCC, ao mostrar que o PC pode ser assimilado em práticas educativas que perpassam por todas as área do conhecimento. O mapa também expõe a categoria sobre Pensamento Computacional, pontuando a identificação desta competência nas práticas, junto com temas/assuntos específicos da área de Computação (Ciência da Computação) que atuaram estritamente como um dos Meios de Condução, para engajar os alunos durante as atividades. O Pensamento Computacional também exibe uma relação direta com o Objetivo / Problema, retratando a necessidade de se desenvolver e/ou aplicar habilidades do raciocínio computacional, por parte do aluno, para solucionar a problemática envolvida na atividade.

Embasado nos argumentos que explicam sobre a rede conceitual, a Figura 5 ilustra os três Formatos de Atividades de Ensino (FAE) identificados a partir das 26 práticas analisadas por meio da Teoria Fundamentada. Pode-se notar que o PC está presente de duas 
VII Congresso Brasileiro de Informática na Educação (CBIE 2018)

Anais do XXIV Workshop de Informática na Escola (WIE 2018)

formas nas FAEs: na área mais interna, quando é trabalhado junto ao objetivo (problema) da atividade; e na seta externa quando é usado como método nas atividades.
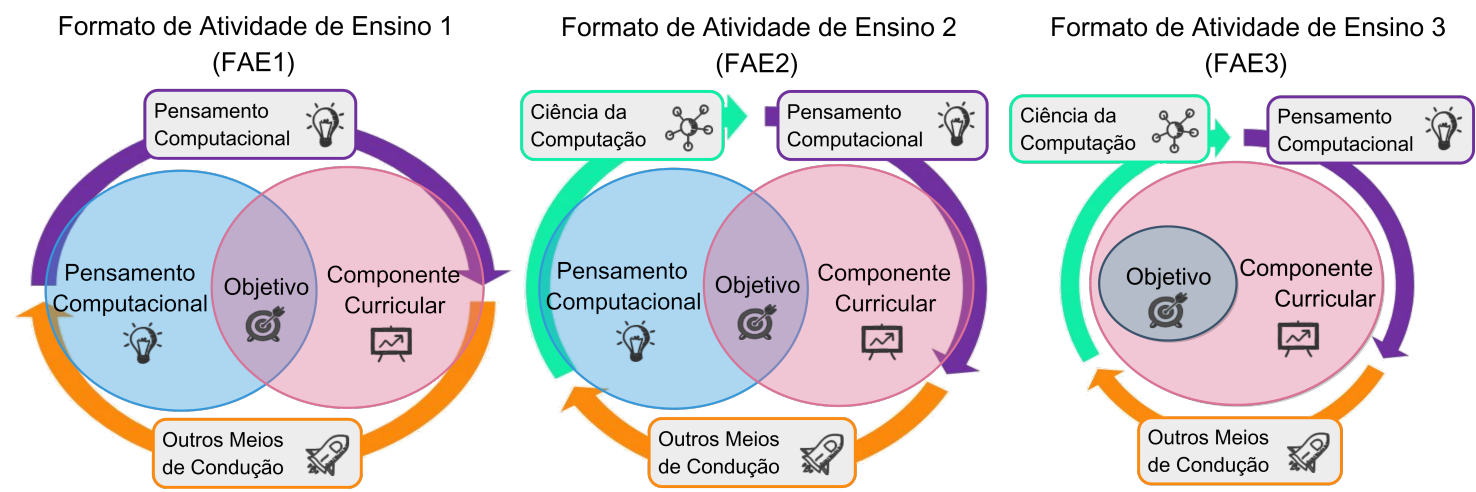

Figura 5. Diagramas dos Formatos das Atividades de Ensino identificados.

A reflexão sobre os resultados obtidos na análise qualitativa mostram que os professores utilizaram o PC como método em todas as atividades de ensino, sempre com apoio de outros meios de condução. Além disso, o alvo/objetivo das atividades de ensino abordam sempre um componente curricular como parte da problemática, mas nem sempre o desenvolvimento de alguma habilidade do PC foi necessário para alcançar esse objetivo. Nota-se também que no FAE2 e no FAE3, as atividades aplicaram um terceiro meio de condução nas práticas de ensino relacionado aos temas/assuntos da Ciência da Computação. Atividades que utilizaram de um tema ou assunto derivado da Computação não tiveram como pretensão a avaliação desse conteúdo, sendo somente incorporada como uma abordagem para desenvolver o raciocínio computacional.

\section{Respostas às Perguntas de Pesquisa}

Para responder cada questão de pesquisa, foram delineadas duas hipóteses (H1 e H2), sendo a primeira baseada nas características mais frequentes identificadas nas práticas de ensino, enquanto que a segunda aponta para particularidades de menor incidência nas atividades. A obtenção de tais hipóteses em ambas as questões apoiaram-se também em duas categorias principais reconhecidas no mapa conceitual da Figura 4, sendo eles o Pensamento Computacional (PC) e o Meios de Condução.

A confirmação de cada hipótese foi feita pela correlação de existência em um ou mais Formatos de Atividades de Ensino (FAE) apresentados na Figura 5. A Tabela 1 indica quais são os FAEs que atendem a cada uma das hipóteses. Esses resultados mostram que, enquanto existe a presença do PC como mecanismo de condução em todos os formatos, apenas os formatos FAE1 e FAE2 abordaram essa competência de maneira integrada a um componente curricular. Por outro lado, todos os casos dependeram de um segundo elemento de conduta da atividade, como destacado na H1 da P2. Esses Outros Meios de Condução são caracterizados por artefatos, insumos, procedimentos e/ou estratégias usados(as) nas práticas, como a Gamificação e a Cultura Maker $^{3}$.

\footnotetext{
${ }^{2}$ Uso de mecânicas, estratégias e elementos recorrentes nos jogos, mas aplicados fora do contexto dos games [de Melo Reis et al. 2017].

${ }^{3}$ Relaciona-se à aprendizagem "mão na massa”, em que o aprendiz combina tecnologias e conhecimentos para a construção de projetos e para a resolução de problemas [Raabe et al. 2017].
} 
VII Congresso Brasileiro de Informática na Educação (CBIE 2018)

Anais do XXIV Workshop de Informática na Escola (WIE 2018)

Tabela 1. Relações entre hipóteses e formatos das atividades

\begin{tabular}{|l|l|c|c|c|}
\hline \multirow{5}{*}{$\mathbf{P 1}$} & $\begin{array}{l}\text { H1: O Pensamento Computacional é aplicado como } \\
\text { método e objetivo na Atividade de Ensino. }\end{array}$ & $\checkmark$ & $\checkmark$ & \\
\hline & $\begin{array}{l}\text { H2: O Pensamento Computacional é aplicado somente } \\
\text { como método da Atividade de Ensino. }\end{array}$ & & & $\checkmark$ \\
\hline \multirow{2}{*}{$\mathbf{P 2}$} & $\begin{array}{l}\text { H1: O Pensamento Computacional é tratado como método } \\
\text { da Atividade de Ensino e vinculado a outros meios de } \\
\text { condução incorporados à prática. }\end{array}$ & $\checkmark$ & $\checkmark$ & $\checkmark$ \\
\hline $\begin{array}{l}\text { H2: O Pensamento Computacional é tratado como método } \\
\text { da Atividade de Ensino e pode ser vinculado aos temas da } \\
\text { Ciência da Computação. }\end{array}$ & $\checkmark$ & $\checkmark$ & $\checkmark$ \\
\hline
\end{tabular}

\section{Considerações Finais}

A oferta de formações continuadas que esclareçam como incorporar o raciocínio computacional de maneira interdisciplinar nas práticas de ensino, são ações que ainda dependem, em maior número, das universidades. Cursos direcionados a essa temática e em diferentes formatos, como presencial [Silva et al. 2017], na modalidade EAD [Barcelos et al. 2016] ou semipresencial, necessitam de abordagens práticas para envolver positivamente os docentes e, em especial, fazerem parte de intervenções constantes do governo.

Este artigo apresentou um estudo de caso sobre como é possível inserir o Pensamento Computacional em atividades de ensino por parte dos professores do EFI. Os resultados da análise qualitativa revelaram que mesmo com dificuldades desses profissionais (como o desconhecimento sobre PC e aspectos de infraestrutura das escolas), eles conseguiram elaborar e aplicar práticas que estimulassem essa competência em seus discentes, de acordo com a sua realidade institucional.

Dos Formatos de Atividade de Ensino (FAE) identificados, foi possível reconhecer que o PC, apoiado em alguma outra estratégia, é facilmente inserido como meio de condução nas práticas de ensino. Quando o PC é um dos objetivos da atividade, é necessário que o professor tenha segurança na compreensão desses conceitos provocados pela problemática da atividade, para que seja possível o desenvolvimento de tais habilidade de forma apropriada.

Como trabalhos futuros, o FAE2 foi selecionado para uma validação e apuração deste modelo, baseado na oferta de duas turmas de uma nova formação continuada, realizada durante o primeiro semestre de 2018. É previsto também um estudo mais aprofundado sobre as estratégias de ensino mais propícias para serem aplicadas em práticas que estimulem o Pensamento Computacional nas crianças. Agradecimentos à CAPES, ao Prof. Dr. Carlos Henrique da Silva Santos (IFSP Câmpus Itapetininga), e às alunas Letícia Mara Berto e Isabella Soares de Lima, membros do C2Y!.

\section{Referências}

Bandeira-de Mello, R. and Cunha, C. J. (2003). Operacionalizando o método da grounded theory nas pesquisas em estratégia: Técnicas e procedimentos de análise com apoio do software atlas/ti. Associação Nacional de Pós-Graduação e Pesquisa em Administração. 1-18. 
VII Congresso Brasileiro de Informática na Educação (CBIE 2018)

Anais do XXIV Workshop de Informática na Escola (WIE 2018)

Barcelos, T., Bortoletto, R., and Andrioli, M. (2016). Formação online para o desenvolvimento do pensamento computacional em professores de matemática. In Workshop de Ensino em Pensamento Computacional, Algoritmos e Programação, 1228-1237.

Charmaz, K. (2009). A construção da Teoria Fundamentada: Guia Prático para Análise Qualitativa. Bookman Editora.

de Melo Reis, F., Cristiano, F., Martins, D., and da Rocha, P. (2017). Pensamento computacional: Uma proposta de ensino com estratégias diversificadas para crianças do ensino fundamental. In Workshop de Informática na Escola, 638-647.

Ferreira, A. C. C., Melhor, A., Barreto, J. d. S., de Paiva, L. F., and Matos, E. (2015). Experiência prática interdisciplinar do raciocínio computacional em atividades de computação desplugada na educação básica. In Workshop de Informática na Escola, 256-265.

França, R. and Tedesco, P. (2015). Desafios e oportunidades ao ensino do pensamento computacional na educação básica no brasil. In Workshop de Ensino em Pensamento Computacional, Algoritmos e Programação, 1464-1473.

Kalelioglu, F., Gülbahar, Y., and Kukul, V. (2016). A framework for computational thinking based on a systematic research review. Baltic Journal of Modern Computing, 4(3). 583-596.

Lazar, J., Feng, J. H., and Hochheiser, H. (2017). Research Methods in Human-Computer Interaction. 2 edition.

Leite, M. and da Silva, S. F. (2017). Redimensionamento da computação em processo de ensino na educação básica: O pensamento computacional, o universo e a cultura digital. In Workshop da Licenciatura em Computação, 804-813.

Martinelli, S. R., Zaina, L. A. M., and Sakata, T. C. (2018). O desenvolvimento do Pensamento Computacional em crianças por meio de Atividades de Ensino mediadas pelo Professor do Ensino Fundamental I: Um Estudo de Caso. Universidade Federal de São Carlos (UFSCar Sorocaba). Relatório Técnico n ${ }^{0}$ 004/2018.

Raabe, A., Metzger, J., Gomes, E., Vieira, M. V., Santana, A. L. M., Souza, F. T., Ramos, G., and Cucco, L. (2017). Características do pensamento computacional desenvolvidas em aprendizes do ensino médio por meio de atividades makers. In Workshop de Informática na Escola, 145-154.

Silva, V., da Silva, L. L., and França, R. (2017). Pensamento computacional na formação de professores: experiências e desafios encontrados no ensino da computação em escolas públicas. In Workshop de Informática na Escola, 805-814.

Valente, J. A. (2016). Integração do pensamento computacional no currículo da educação básica: Diferentes estratégias usadas e questões de formação de professores e avaliação do aluno. In Revista e-Curriculum, 864-897.

Wing, J. M. (2006). Computational thinking. Communications of the ACM, 49(3):33-35.

Wing, J. M. (2014). Computational thinking benefits society. 40th Anniversary Blog of Social Issues in Computing. 